\title{
Subspace-based frequency estimation of sinusoidal signals in alpha-stable noise ${ }^{\text {is }}$
}

\author{
Mustafa A. Altınkaya ${ }^{\mathrm{a}, *}$, Hakan Deliç, Bülent Sankur ${ }^{\mathrm{b}}$, Emin Anarım$^{\mathrm{b}}$ \\ ${ }^{a}$ Department of Electrical and Electronics Engineering, İzmir Institute of Technology, Gülbahçe Köyü, Urla 35437 Izmir, Turkey \\ ${ }^{\mathrm{b}}$ Signal and Image Processing Laboratory (BUSIM), Department of Electrical and Electronics Engineering, Boğaziçi University, \\ Bebek 80815 Istanbul, Turkey
}

Received 30 April 2001; received in revised form 12 September 2001

\begin{abstract}
In the frequency estimation of sinusoidal signals observed in impulsive noise environments, techniques based on Gaussian noise assumption are unsuccessful. One possible way to find better estimates is to model the noise as an alpha-stable process and to use the fractional lower order statistics (FLOS) of the data to estimate the signal parameters. In this work, we propose a FLOS-based statistical average, the generalized covariation coefficient (GCC). The GCCs of multiple sinusoids for unity moment order in $\mathrm{S} \alpha \mathrm{S}$ noise attain the same form as the covariance expressions of multiple sinusoids in white Gaussian noise. The subspace-based frequency estimators FLOS-multiple signal classification (MUSIC) and FLOSBartlett are applied to the GCC matrix of the data. On the other hand, we show that the multiple sinusoids in $\mathrm{S} \alpha \mathrm{S}$ noise can also be modeled as a stable autoregressive moving average process approximated by a higher order stable autoregressive (AR) process. Using the GCCs of the data, we obtain FLOS versions of Tufts-Kumaresan (TK) and minimum norm (MN) estimators, which are based on the AR model. The simulation results show that techniques employing lower order statistics are superior to their second-order statistics (SOS)-based counterparts, especially when the noise exhibits a strong impulsive attitude. Among the estimators, FLOS-MUSIC shows a robust performance. It behaves comparably to MUSIC in non-impulsive noise environments, and both in impulsive and non-impulsive high-resolution scenarios. Furthermore, it offers a significant advantage at relatively high levels of impulsive noise contamination for distantly located sinusoidal frequencies.
\end{abstract}

(c) 2002 Elsevier Science B.V. All rights reserved.

Keywords: Frequency estimation; Parameter estimation; Alpha-stable noise; Impulsive noise; Subspace method

\section{Introduction}

Most of the work on the frequency estimation problem assumes that the additive noise has Gaussian distribution. This is partly because of the desirable properties that the Gaussian model possesses, which allow

\footnotetext{
is A preliminary version of this paper was presented in the 8th IEEE Signal Processing Workshop on Statistical Signal and Array Processing, Corfu, Greece, June 24-26, 1996.

* Corresponding author.

E-mail addresses: altink@likya.iyte.edu.tr (M.A. Altınkaya), delic@boun.edu.tr (H. Deliç), sankur@boun.edu.tr (B. Sankur), anarim@boun.edu.tr (E. Anarım).
} 
for simplification of the theoretical work and decrease the computational complexity in signal parameter estimation. As long as the acting noise distribution can fit approximately to a Gaussian model, in particular for the tails of the distribution, one can obtain good estimators with the Gaussian noise assumption. But if the noise process belongs to a non-Gaussian, especially a heavy-tailed distribution class, or when the noise is of impulsive nature, parameter estimators that are based on the Gaussian assumption break down. Applications such as cellular telephony (due to atmospheric disturbances) and underwater acoustics (due to cracking ice), for example, are known to take place in additive noise displaying impulsive behavior $[10,12]$.

Impulsive noise processes can be modeled using stable distributions. If a signal can be thought of as the sum of a large number of independent and identically distributed random variables, the limiting distribution is in the class of stable distributions according to the generalized central limit theorem [12]. Stable distributions cover the Gaussian distribution in the limit.

Parameter estimation in the presence of alpha-stable noise involves challenges that demand certain deviations from classical signal processing. The optimal maximum likelihood solutions are nearly impossible to implement [17]. Approximations to the likelihood function that utilize the estimates of the alpha-stable distribution parameters also result in complex structures. In [7], it is shown that the blind estimation of the parameters of an autoregressive moving average (ARMA) model driven by an alpha-stable process is feasible. However, the blind algorithm requires intensive computation and possible phase unwrapping, while consistency is through convergence in probability [15]. Work on less complex, suboptimal alternatives includes the parameter estimation of a deterministic signal by applying the $M$-estimate theory [3].

Other related work include Tsakalides and Nikias' application of robust covariation-based multiple signal classification (ROC-MUSIC), which is a noise subspace method, to the direction of arrival estimation problem [16]. More recently, Liu and Mendel [6] investigated the same problem for those signals that consist of circular signals in symmetric alpha-stable $(\mathrm{S} \alpha \mathrm{S})$ noise by just using the fractional lower order moments (FLOMs).

The related problem of frequency estimation of sinusoids in impulsive noise as modeled by the alpha-stable distributions has not been tackled in literature. Considering the potential issues accompanying blind schemes (described in the previous paragraph), as a first step, we evaluate the subspace-based frequency estimators in this paper. The subspace techniques attain statistical performance close to the accurate non-linear least-squares frequency estimator in Gaussian noise without the multidimensional search required by the latter [13]. Moreover, they provide very good separation between closely located tones $[4,13]$.

In this work, we consider the particular problem of frequency estimation of multiple sinusoids in $\mathrm{S} \alpha \mathrm{S}$ noise. In order to use the possible solutions adaptable from the vast amount of second-order statistics (SOS)-based frequency estimation methods, we either model the $\mathrm{S} \alpha \mathrm{S}$ noise-contaminated signal as a stable ARMA process, or just estimate the fractional lower order statistics (FLOS) of the data. For the former class of methods, we utilize the fact that any sinusoidal signal in white noise can be modeled as an ARMA process with equal autoregressive (AR) and moving average (MA) orders [4,11], and approximate this ARMA system with an AR system of higher order.

For the methods exploiting the structure of the covariance matrix such as MUSIC or principal componentBartlett (PC-Bartlett), we apply them to the FLOS of data, similar to the approach adopted by Liu and Mendel [6]. By applying the same expectation operation of the covariation coefficient, we propose the generalized covariation coefficient (GCC) concept. Thus, we unify the FLOS estimation process for both type of the methods. We show that the GCCs for the randomly phased sinusoids in $\mathrm{S} \alpha \mathrm{S}$ noise are composed of a sum of cosinusoids and a noise term whose constant positive amplitudes depend non-linearly on the particular $\mathrm{S} \alpha \mathrm{S}$ probability density function (pdf) and on the relative generalized signal-to-noise ratios (GSNR) for the unity moment order. Motivated by the success of subspace methods in white Gaussian noise, we then proceed to apply MUSIC, PC-Bartlett, Tufts-Kumaresan (TK) and minimum norm (MN) methods to FLOS of sinusoids embedded in alpha-stable noise. Rigorous simulation studies indicate that the GCC-based frequency estimators outperform the traditional estimators which work with SOS. 
In Section 2, the $\mathrm{S} \alpha \mathrm{S}$ distributions are briefly discussed. In Section 3, the application of FLOMs to frequency estimation problem is presented. Section 4 covers the results of the simulation experiments. Finally, conclusions are in Section 5.

Regarding the notation used in the paper, we adopt uppercase letters for random variables and corresponding lowercase letters for the realizations of these random variables, respectively.

\section{2. $\mathrm{S} \alpha \mathrm{S}$ distributions}

An important subclass of stable distributions are $S \alpha S$ distributions. The characteristic function of $S \alpha S$ variables is given by

$$
\phi(\omega)=\exp \left\{\mathrm{j} \delta \omega-\gamma|\omega|^{\alpha}\right\}
$$

where $\alpha$ is the characteristic exponent $(0<\alpha \leqslant 2)$ describing the thickness of the tails, $\delta$ is the location parameter $(-\infty<\delta<\infty)$ defining the center of the distribution, and $\gamma$ is the dispersion $(\gamma>0)$, which plays an analogous role as the variance does for a second-order process and determines the scale of the distribution. Gaussian and Cauchy distributions are members of the $S \alpha S$ family for $\alpha=2$ and 1, respectively. Without loss of generality, the location parameter $\delta$ can be set to zero, leading to the characteristic function

$$
\phi(\omega)=\exp \left\{-\gamma|\omega|^{\alpha}\right\} .
$$

For $\mathrm{S} \alpha \mathrm{S}$ processes, only the moments of order $p<\alpha$ exist, and consequently, the estimation methods based on SOS of the data cannot be applied. One solution is to utilize the FLOS of the process. The $p$ th order moment of a $\mathrm{S} \alpha \mathrm{S}$ random variable $X$ is given by [10]

$$
E\left(|X|^{p}\right)=C(p, \alpha) \gamma_{x}^{p / \alpha}, \quad 0<p<\alpha,
$$

where $\gamma_{x}$ is the dispersion of random variable $X$, and

$$
C(p, \alpha)=\frac{2^{p+1} \Gamma((p+1) / 2) \Gamma(-p / \alpha)}{\alpha \sqrt{\pi} \Gamma(-p / 2)} .
$$

Note that $C(p, \alpha)$ does not depend on $X . \Gamma$ is the usual gamma function defined as

$$
\Gamma(x)=\int_{0}^{\infty} t^{x-1} \mathrm{e}^{-t} \mathrm{~d} t .
$$

In the FLOS-based estimation methods, the so-called covariations [9] of two random variables can be utilized. The covariation of two jointly $\mathrm{S} \alpha \mathrm{S}$ real random variables with dispersions $\gamma_{x}$ and $\gamma_{y}$ are given as

$$
[X, Y]_{\alpha}=\frac{E\left[X Y^{\langle p-1\rangle}\right]}{E\left[|Y|^{p}\right]} \gamma_{y}
$$

where $\gamma_{y}=[Y, Y]_{\alpha}$ is the dispersion of random variable $Y$ and $Y^{\langle p-1\rangle}=|Y|^{p-1} \operatorname{signum}(Y)$ with

$$
\operatorname{signum}(Y)=\left\{\begin{aligned}
1 & \text { for } Y>0, \\
0 & \text { for } Y=0, \\
-1 & \text { for } Y<0 .
\end{aligned}\right.
$$


The above definition of covariation is independent of $p$ as long as $1 \leqslant p<\alpha$ [1]. Another useful FLOS is the covariation coefficient of those jointly $\mathrm{S} \alpha \mathrm{S}$ real random variables which is given as

$$
\lambda_{X, Y}=\frac{[X, Y]_{\alpha}}{[Y, Y]_{\alpha}}=\frac{E\left[X Y^{\langle p-1\rangle}\right]}{E\left[|Y|^{p}\right]}, \quad 1 \leqslant p<\alpha .
$$

Notice that the covariation coefficient $\lambda_{X, Y}$ is equal to the covariation $[X, Y]_{\alpha}$ scaled by $\gamma_{y}$, which is a constant.

\section{The frequency estimation problem}

In the frequency estimation problem, the signal model considered consists of multiple sinusoids

$$
s_{n}=\sum_{k=1}^{K} A_{k} \sin \left\{\omega_{k} n+\theta_{k}\right\}
$$

observed in additive $\mathrm{S} \alpha \mathrm{S}$ noise

$$
x_{n}=s_{n}+z_{n}, \quad n=1, \ldots, N,
$$

where $A_{k}$ is the amplitude, $\omega_{k}$ is the angular frequency, and $\theta_{k}$ is the phase of the $k$ th real sinusoid. $\left\{A_{k}, k=\right.$ $1, \ldots, K\}$ and $\left\{\omega_{k}, k=1, \ldots, K\right\}$ are unknown real constants, whereas $\left\{\theta_{k}, k=1, \ldots, K\right\}$ are assumed to be realizations of random variables, distributed uniformly and independently over $[0,2 \pi) . K$ is the number of sinusoids, and $N$ is the sample size. $x_{n}$ and $z_{n}$ are realizations of the observation sequence $X_{n}$ and the independent and identically distributed $\mathrm{S} \alpha \mathrm{S}$ noise sequence $Z_{n}$, respectively.

It is well known that $s_{n}$ obeys the following AR difference equation [5,14]:

$$
B\left(q^{-1}\right) s_{n}=0,
$$

where $q^{-1}$ denotes the unit delay operator $\left(q^{-1} s_{n}=s_{n-1}\right)$ and $B\left(q^{-1}\right)$ is a polynomial of degree $2 K$ given by

$$
B\left(q^{-1}\right)=1+b_{1} q^{-1}+\cdots+b_{2 K} q^{-2 K}=\prod_{m=1}^{K}\left(1-2 \cos \omega_{m} q^{-1}+q^{-2}\right) .
$$

From Eqs. (9) and (12), one can deduce that $x_{n}$ obeys the following ARMA difference equation:

$$
B\left(q^{-1}\right) x_{n}=B\left(q^{-1}\right) z_{n}
$$

It is possible to approximate the above stable ARMA process with a stable AR process of order $M$ where $M \geqslant 2 K$. Experiments with second-order AR processes in literature show that as model order increases, the AR model approximation becomes better, which is a manifestation of the Kolmogorov theorem [4]. When the noise dispersion is small, an AR model order of $M=2 \mathrm{~K}$ might be appropriate but as the noise dispersion increases, a higher $M$ is required for satisfactory approximation.

Thus, the observation sequence can be modeled as a stable AR process

$$
X_{n}=a_{1} X_{n-1}+\cdots+a_{M} X_{n-M}+b_{0} Z_{n},
$$

where the model order $M$ of the AR model for the noisy signal should be selected higher than $2 K$ in order to allow for sufficient additional subspace dimension for the noise component as in the additive Gaussian noise 
case. This leads to the generalized Yule-Walker equation when $X_{n-m}$ is given as [12]

$$
\begin{aligned}
& E\left[X_{n} \mid X_{n-m}\right]=a_{1} E\left[X_{n-1} \mid X_{n-m}\right]+\cdots+a_{M} E\left[X_{n-M} \mid X_{n-m}\right], \\
& E\left[X_{n+l} \mid X_{n}\right]=\tilde{\lambda}(l) X_{n},
\end{aligned}
$$

where $m=1, \ldots, M$. If $\tilde{\lambda}(l)$ denotes the covariation coefficient of $X_{n+l}$ with $X_{n}$, one can find the AR parameters by solving the following linear set of equations:

$$
\tilde{\mathbf{C}}_{\mathbf{x}} \mathbf{a}=\tilde{\lambda}
$$

with

$$
\tilde{\mathbf{C}}_{\mathbf{x}}=\left[\begin{array}{cccc}
\tilde{\lambda}(0) & \tilde{\lambda}(-1) & \ldots & \tilde{\lambda}(1-M) \\
\tilde{\lambda}(1) & \tilde{\lambda}(0) & \ldots & \tilde{\lambda}(2-M) \\
\vdots & \vdots & \vdots & \vdots \\
\tilde{\lambda}(M-1) & \tilde{\lambda}(M-2) & \cdots & \tilde{\lambda}(0)
\end{array}\right], \quad \mathbf{a}=\left[\begin{array}{c}
a_{1} \\
a_{2} \\
\vdots \\
a_{M}
\end{array}\right], \quad \tilde{\lambda}=\left[\begin{array}{c}
\tilde{\lambda}(1) \\
\tilde{\lambda}(2) \\
\vdots \\
\tilde{\lambda}(M)
\end{array}\right]
$$

The covariation coefficient matrix $\tilde{\mathbf{C}}_{\mathbf{x}}$ defined in Eq. (17) for alpha-stable processes has the same meaning as that of the covariance matrix for Gaussian processes [10]. As one performs eigen-decomposition of the covariation coefficient matrix, the larger eigenvalues will correspond to signal subspace eigenvectors and the remaining eigenvectors will constitute the noise subspace. Thus, one can perform eigenanalysis on the covariation coefficient matrix, and then apply a suitable noise subspace or a signal subspace technique to estimate the parameters. Note that the covariation coefficient matrix is not symmetric. This makes the eigenanalysis more complicated, and renders many of the subspace-based parameter estimation techniques developed for Gaussian processes unsuitable for the general alpha-stable processes. One should also note that the mentioned properties of the covariation coefficient matrix are shared by the covariation matrix which is a scaled version of the covariation coefficient matrix.

\section{1. $G C C$}

In this subsection, we apply the FLOS-based expectation operation in Eq. (8) which is used to calculate the covariation coefficient, to our randomly phased sinusoids in $\mathrm{S} \alpha \mathrm{S}$ noise model defined in Eqs. (9) and (10). Specifically, we define

$$
\lambda_{X_{n}, X_{l}}(p)=\frac{E\left[X_{n} X_{l}^{\langle p-1\rangle}\right]}{E\left[\left|X_{l}\right|^{p}\right]}, \quad n, l=1, \ldots, M, 0<p<\alpha,
$$

as the GCC, where no assumptions are made on the pdf of the samples of the random process $X$. Inserting Eqs. (9) and (10) into Eq. (18),

$$
\begin{aligned}
\lambda_{X_{n}, X_{l}}(p)= & E\left\{\left(\sum_{i=1}^{K} A_{i} \sin \left\{\omega_{i} n+\Theta_{i}\right\}+Z_{n}\right)\left|\sum_{j=1}^{K} A_{j} \sin \left\{\omega_{j} l+\Theta_{j}\right\}+Z_{l}\right|^{p-1}\right. \\
& \left.\times \operatorname{signum}\left(\sum_{j=1}^{K} A_{j} \sin \left\{\omega_{j} l+\Theta_{j}\right\}+Z_{l}\right)\right\}\left(E\left|\sum_{j=1}^{K} A_{j} \sin \left\{\omega_{j} l+\Theta_{j}\right\}+Z_{l}\right|^{p}\right)^{-1} .
\end{aligned}
$$

Because of the non-linearity introduced by the second and third components in the expectation operation, it is not possible to obtain a closed form solution for general $p$ values. But the choice $p=1$ brings interesting 
simplifications, and the equation reduces to

$$
\begin{aligned}
\left.\lambda_{X_{n}, X_{l}}(p)\right|_{p=1}= & E\left\{\left(\sum_{i=1}^{K} A_{i} \sin \left\{\omega_{i} n+\Theta_{i}\right\}+Z_{n}\right) \operatorname{signum}\left(\sum_{j=1}^{K} A_{j} \sin \left\{\omega_{j} l+\Theta_{j}\right\}+Z_{l}\right)\right\} \\
& \times\left(E\left|\sum_{j=1}^{K} A_{j} \sin \left\{\omega_{j} l+\Theta_{j}\right\}+Z_{l}\right|\right)^{-1} .
\end{aligned}
$$

Utilizing the above definition, the GCCs of a randomly phased sinusoid observed in $\mathrm{S} \alpha \mathrm{S}$ noise are given as

$$
\left.\lambda_{X_{n}, X_{l}}(p)\right|_{p=1}=\zeta_{1} \cos \left\{\omega_{1}(n-l)\right\}+P_{z_{l}}^{(1)} \delta_{n l},
$$

where $\zeta_{1}$ and $P_{z_{l}}^{(1)}$ are positive real constants depending on the particular $\mathrm{S} \alpha \mathrm{S}$ pdf and $\delta_{n l}$ is the Kronecker delta. (See Appendix A for the derivation of this equation.) The GCCs of two randomly phased sinusoids observed in $\mathrm{S} \alpha \mathrm{S}$ noise are obtained in a similar form given by

$$
\left.\lambda_{X_{n}, X_{l}}(p)\right|_{p=1}=\sum_{i=1}^{2} \xi_{i} \cos \left\{\omega_{i}(n-l)\right\}+P_{z_{l}}^{(2)} \delta_{n l},
$$

where $\xi_{1}, \xi_{2}$ and $P_{z_{l}}^{(2)}$ are also positive real constants depending on the particular $\mathrm{S} \alpha \mathrm{S}$ pdf. (The derivation of this equation is given in Appendix B.)

Using the method described in Appendices A and B, one can find that for the moment order $p=1$, the GCCs of $K$ randomly phased sinusoids observed in $\mathrm{S} \alpha \mathrm{S}$ noise can be expressed as

$$
\left.\lambda_{X_{n}, X_{l}}(p)\right|_{p=1}=\sum_{k=1}^{K} \varrho_{k} \cos \left\{\omega_{k}(n-l)\right\}+P_{z_{l}}^{(K)} \delta_{n l},
$$

where $\left\{\varrho_{k}, k=1, \ldots, K\right\}$ are positive real constants depending non-linearly on the $\alpha$ of the particular $\mathrm{S} \alpha \mathrm{S}$ noise contaminating the sinusoidal signals, and on the magnitudes of the sinusoids as demonstrated in the Appendices for single- and two-sinusoid cases.

This is a significant result since it shows that many techniques developed to exploit the eigenstructure of the covariance matrix for the sinusoidal parameter estimation problems in Gaussian noise environments are also applicable to the FLOS of the sinusoids embedded in $\mathrm{S} \alpha \mathrm{S}$ noise, at least for the case of $p=1$. Note that the $\left\{\varrho_{k}, k=1, \ldots, K\right\}$ can be factored out to two components where one of them linearly depends on the corresponding sinusoid and the other component depends non-linearly on all the sinusoidal signal powers and $\alpha$ as described in Appendix B.

Eq. (23) is similar to the well-known SOS covariance equation of sinusoids observed in white Gaussian noise, where the magnitudes of the cosinusoids and the noise component in the covariance expression are linearly dependent on the powers of the sinusoids and the noise [11]. Thus, we can apply the same eigendecomposition procedure to our GCC matrix

$$
\mathbf{C}_{\mathbf{x}}^{(1)}=\mathbf{C}_{\mathbf{s}}^{(1)}+P_{z_{l}}^{(K)} \mathbf{I},
$$

where

$$
\mathbf{C}_{\mathbf{s}}^{(1)}=\sum_{k=1}^{K} \frac{\varrho_{k}}{2}\left\{\mathbf{s}_{k} \mathbf{s}_{k}^{H}+\mathbf{s}_{k}^{*} \mathbf{s}_{k}^{\mathrm{T}}\right\}
$$

with $\mathbf{s}_{k}=\left[1 \exp \left\{\mathrm{j} \omega_{k}\right\} \cdots \exp \left\{\mathrm{j} \omega_{k}(M-1)\right\}\right]$. The superscripts $H, *$ and $\mathrm{T}$ denote the conjugate transpose, conjugate and the transpose operations, respectively, and $\mathbf{I}$ is the $M \times M$ identity matrix. The rank of $\mathbf{C}_{\mathbf{s}}^{(1)}$ is $2 K<M$. But $\mathbf{C}_{\mathbf{x}}^{(1)}$ is full rank, since $P_{z_{l}}^{(K)} \mathbf{I}$ is full rank. 


\subsection{FLOS-MUSIC and FLOS-Bartlett frequency estimators}

The subspace techniques exploit the geometric properties of the measurement signal and noise characteristics to estimate the desired parameters [4,8]. One of the most popular ones among these is the MUSIC algorithm.

The usual SOS-based MUSIC algorithm utilizes the sample covariance matrix given by

$$
\hat{\mathbf{R}}_{(N, M)}=\frac{1}{N-M+1} \sum_{k=1}^{N-M+1} \mathbf{x}_{k} \mathbf{x}_{k}^{H},
$$

where

$$
\mathbf{x}_{k}=\left[\begin{array}{llll}
x_{k} & x_{k+1} & \cdots & x_{k+M-1}
\end{array}\right]^{\mathrm{T}} .
$$

One can obtain the frequency estimates of a FLOS-based MUSIC, which we shall call FLOS-MUSIC [16], utilizing the sample GCC matrix $\hat{\mathbf{C}}_{\mathbf{x}}^{(p)}$ instead of the sample covariance matrix, where the $(n, l)$ th element of the $\hat{\mathbf{C}}_{\mathbf{x}}^{(p)}$ is given by

$$
\hat{\mathbf{C}}_{\mathbf{x}}^{(p)}=\left\{\hat{\lambda}_{p}(n, l), n, l=1, \ldots, M\right\}, \quad 0<p<\alpha
$$

with the sample GCCs

$$
\hat{\lambda}_{p}(n, l)=\hat{\lambda}_{X_{n}, X_{l}}(p)=\frac{\sum_{i=1}^{N-M+1} X_{n+i-1}\left|X_{l+i-1}\right|^{p-1} \operatorname{signum}\left(X_{l+i-1}\right)}{\sum_{i=1}^{N-M+1}\left|X_{l+i-1}\right|^{p}}, \quad n, l=1, \ldots, M,
$$

defined for moment order $p \in(0, \alpha)$. M denotes the number of columns of the square sample GCC matrix. Note that the modified FLOM (MFLOM) estimator for jointly $\mathrm{S} \alpha \mathrm{S}$ random variables has the same form of Eq. (29) where MFLOM is defined for moment order $p \in(0.5, \alpha)[16]$.

Performing the eigendecomposition of the sample GCC matrix, the FLOS-MUSIC frequency estimates are found as the peaks of the spectrum given by

$$
\operatorname{FLOS}-\operatorname{MUSIC}(\omega)=\frac{1}{\sum_{i=2 K+1}^{M}\left|\mathbf{d}^{H} \hat{\mathbf{v}}_{i}\right|^{2}},
$$

where $\mathbf{d}$ is the complex sinusoidal vector $\mathbf{d}=[1 \exp \{\mathrm{j} \omega\} \cdots \exp \{\mathrm{j} \omega(M-1)\}]^{\mathrm{T}}$, and $\left\{\hat{\mathbf{v}}_{i}, i=2 K+1, \ldots, M\right\}$ are the estimated column noise subspace eigenvectors corresponding to the smallest $M-2 K$ estimated eigenvalues of the sample GCC matrix.

As in the case of the MUSIC frequency estimator, the SOS-based PC-Bartlett [4] is adapted by using the estimated eigenvectors of the sample GCC matrix instead of the sample covariance matrix. Then the component frequencies are estimated by the peaks of the FLOS-Bartlett spectrum:

$$
\text { FLOS-Bartlett }(\omega)=\frac{1}{M} \sum_{i=1}^{2 K} \hat{\eta}_{i}\left|\mathbf{d}^{H} \hat{\mathbf{v}}_{i}\right|^{2}
$$

where $\hat{\eta}_{i}$ and $\hat{\mathbf{v}}_{i}$ are estimates of the ordered eigenvalues such that $\hat{\eta}_{1} \geqslant \hat{\eta}_{2} \geqslant \cdots \geqslant \hat{\eta}_{M}$, and the corresponding estimated eigenvectors of the $M M$ sample GCC matrix, respectively.

\subsection{FLOS-TK and FLOS-MN frequency estimators}

The SOS-based TK frequency estimator [19] utilizes the principal component eigenvalues and eigenvectors of the sample covariance matrix to find the sinusoidal frequencies. This method can also be called as principal 
component autoregressive method of frequency estimation [4]. The AR coefficients $\hat{\mathbf{a}}$ are obtained as [19]

$$
\hat{\mathbf{a}}=\hat{\mathbf{R}}_{(N-1, M)}^{\#} \hat{\mathbf{r}}_{(N-1, M)}
$$

with

$$
\hat{\mathbf{r}}_{(N-1, M)}=-\frac{1}{N-M} \sum_{k=1}^{N-M} \mathbf{x}_{k+1} x_{k}^{*}
$$

and

$$
\hat{\mathbf{R}}_{(N-1, M)}^{\#}=\sum_{i=1}^{2 K} \frac{1}{\hat{\eta}_{i}} \hat{\mathbf{v}}_{i} \hat{\mathbf{v}}_{i}^{H} .
$$

Here, $\hat{\eta}_{i}$ and $\hat{\mathbf{v}}_{i}$ denote the ordered estimates of eigenvalues and the corresponding estimated eigenvectors, respectively. The TK frequency estimates are obtained as the peaks of the spectrum given by

$$
\operatorname{TK}(\omega)=\frac{1}{\left|1+\mathbf{d}^{H} \hat{\mathbf{a}}\right|^{2}} .
$$

The FLOS-TK frequency estimates are obtained by utilizing the sample GCC matrix $\hat{\mathbf{C}}_{\mathbf{x}}^{(p)}$ and the sample GCC vector $\hat{\lambda}(p)=\left[\hat{\lambda}_{p}(2,1), \hat{\lambda}_{p}(3,2), \ldots, \hat{\lambda}_{p}(M+1, M)\right]^{\mathrm{T}}$ instead of the sample covariance matrix $\hat{\mathbf{R}}_{(N-1, M)}$ and the sample correlation vector $\hat{\mathbf{r}}_{(N-1, M)}$, respectively.

In order to find the $\mathrm{MN}$ frequency estimate, first the eigen-decomposition of $(M+1) \times(M+1)$ sample covariance matrix $\hat{\mathbf{R}}_{(N, M+1)}$ is performed.

$$
\hat{\mathbf{G}}_{\mathbf{s}}^{\prime}=\left[\begin{array}{llll}
\hat{\mathbf{v}}_{1} & \hat{\mathbf{v}}_{2} & \cdots & \hat{\mathbf{v}}_{2 K}
\end{array}\right]=\left[\begin{array}{c}
\hat{\mathbf{g}}_{\mathbf{s}}^{\mathrm{T}} \\
\cdots \\
\hat{\mathbf{G}}_{\mathbf{s}}
\end{array}\right],
$$

where $\hat{\mathbf{g}}_{\mathrm{s}}^{\mathrm{T}}$ is the $1 \times 2 K$ row vector constructed with the first elements of the estimated signal subspace eigenvectors and $\hat{\mathbf{G}}_{\mathbf{s}}$ is the $M \times 2 K$ matrix having the estimated signal subspace eigenvectors except their first rows. Then, the AR coefficients are found as

$$
\hat{\mathbf{a}}=\left(1-\hat{\mathbf{g}}_{\mathbf{s}}^{H} \hat{\mathbf{g}}_{\mathbf{s}}\right)^{-1} \hat{\mathbf{G}}_{\mathbf{s}}^{*} \hat{\mathbf{g}}_{\mathbf{s}}
$$

and the SOS-based MN frequency estimates are obtained as the peaks of the spectrum given by

$$
\operatorname{MN}(\omega)=\frac{1}{\left|1+\mathbf{d}^{H} \hat{\mathbf{a}}\right|^{2}} .
$$

The FLOS-MN frequency estimates are obtained by utilizing the $(M+1) \times(M+1)$ sample GCC matrix $\hat{\mathbf{C}}_{\mathbf{x}}^{(p)}$ instead of the sample covariance matrix $\hat{\mathbf{R}}_{(N, M+1)}$.

\section{Experimental results}

We utilize the FLOS-based MUSIC, Bartlett, TK and MN methods to estimate the sinusoidal frequencies in the single- and two-real sinusoid cases. We apply $\mathrm{S} \alpha \mathrm{S}$ noise sequences with varying $\alpha$ and $\gamma$ parameters. Although the parameters of the alpha-stable process are assumed to be known, they can be estimated a priori via, for instance, the algorithms in [18]. To generate the $\mathrm{S} \alpha \mathrm{S}$ noise process, we employ the method described by Tsihrintzis and Nikias [17], which is a special case of a more general technique encompassing the non-symmetric alpha-stable random variable generation prescribed by Chambers et al. [2]. The moment 
Variance Reduction (dB)
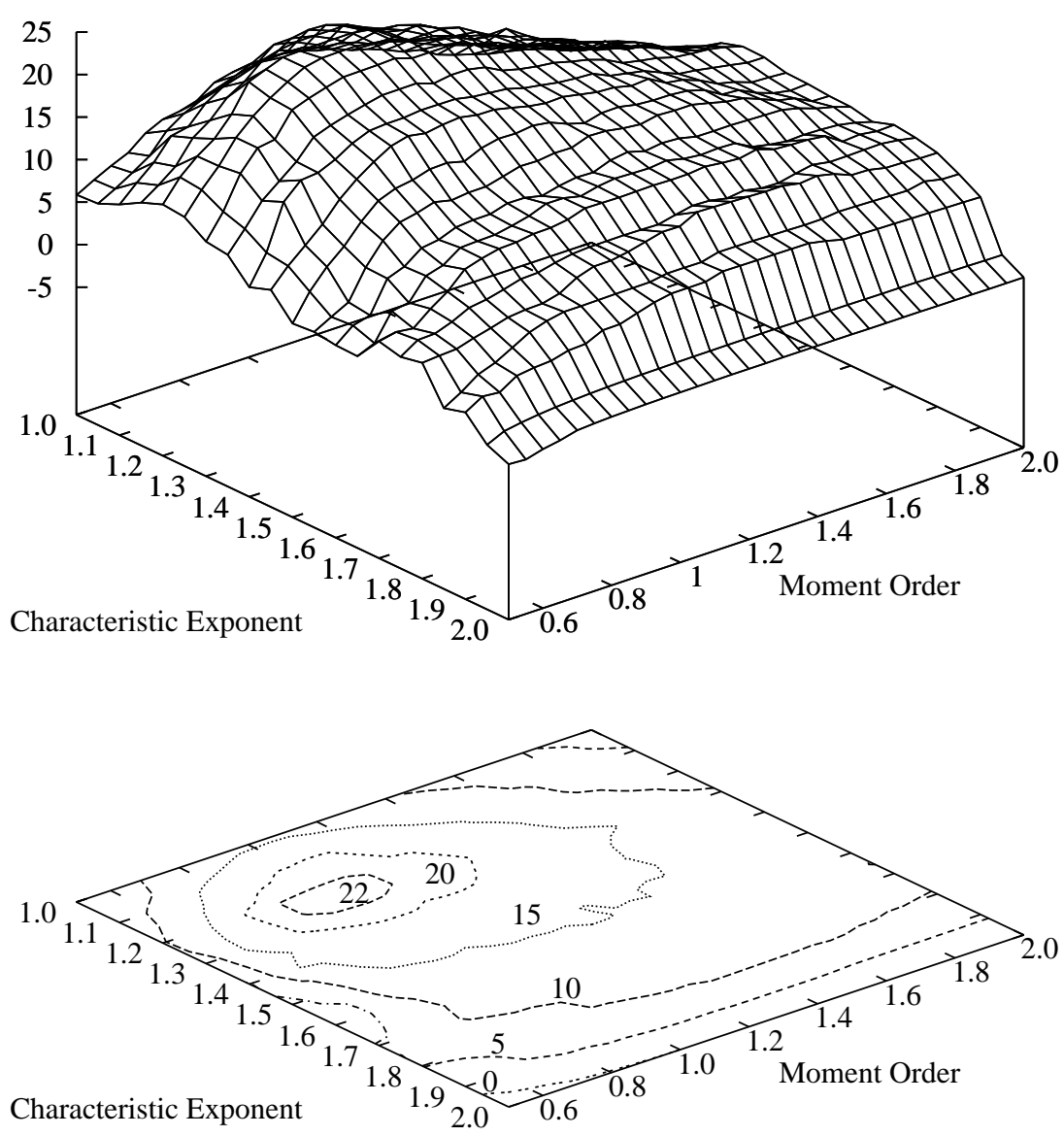

Fig. 1. Variance reduction of FLOS-Bartlett with respect to PC-Bartlett frequency estimator versus GSNR and fractional moment order, averaged on the frequency axis $(M=20, N=50,100$ noise and phase realizations).

frequency order $p$ and the sample size $N$ are equal to 1 and 50, respectively. The AR model order for MNand TK-type estimators is chosen to be 20 and the sample GCC matrix $\hat{\mathbf{C}}_{\mathbf{x}}^{(p)}$ is $20 \times 20$ in the simulations. The generalized SNR [16], GSNR, is defined as: GSNR $=10 \log \left\{\left(\sum_{n=1}^{N}|s(n)|^{2}\right) / \gamma N\right\}$.

\subsection{Single real tone case}

\subsubsection{Dependence of variance reduction upon $\alpha$ and $p$}

In Fig. 1, the variance reduction achieved by FLOS-Bartlett with respect to PC-Bartlett is plotted against $\alpha$ and $p$. The number of Monte Carlo runs is 100 , each with a different noise and phase realization, and GSNR $=10 \mathrm{~dB}$. The gain surface shows that FLOS-Bartlett always demonstrates superior performance with respect to the PC-Bartlett, excluding a small area where GSNR $=0 \mathrm{~dB}$ and $\alpha=2$, i.e., at very low GSNR values and Gaussian noise conditions. As $\alpha$ decreases, the impulsiveness and hence the gain of the FLOS-Bartlett 
increases. The highest gain areas correspond to values of $p \simeq 1$ and $\alpha \simeq 1.3$, which is higher than $22 \mathrm{~dB}$. This obvious superiority of the FLOS-based statistical estimator with respect to its SOS-based counterpart is a consequence of the fact that the SOS of $\alpha$-stable processes are not defined as mentioned in Section 2 . It is also worth noting that a surface defined by the choice of $p=1$ crosses the highest gains for each $\alpha$ value, which implies that $p \simeq 1$ can be a good candidate for computing the GCCs. This behavior is anticipated since the GCC matrix of multiple sinusoids in white noise for $p=1$ attains the form in Eq. (24), which is exploited by the subspace techniques such as FLOS-MUSIC and FLOS-Bartlett in the sinusoidal frequency estimation problem. The deviation from this form caused by other choices for the $p$ value deteriorates the performance of these estimators. The behavior of FLOS-Bartlett is also shared by the noise subspace technique FLOS-MUSIC. Hence, in the remaining simulations we fixed the value of $p$ to one.

\subsubsection{Dependence of variance reduction upon $\alpha$ and GSNR}

Fig. 2 depicts the variance reduction achieved by FLOS-Bartlett with respect to PC-Bartlett, versus $\alpha$ and GSNR. The number of Monte Carlo runs is 100 , each with a different noise and phase realization. The smaller the $\alpha$ value, the higher the gain. Those parts of the surface which exhibit the highest gain belong to $\alpha=1.001$. This gain is approximately more than $20 \mathrm{~dB}$, when GSNR $=10 \mathrm{~dB}$. The surface shows that a performance drop occurs in FLOS-Bartlett with respect to PC-Bartlett, only for the limiting values of $\alpha \rightarrow 2$ and at low GSNR levels. In other words, the FLOS-based subspace techniques can be said to be robust in that they remain superior for a very wide range of $\alpha$ and GSNR values, and this behavior is also shared by the noise subspace technique FLOS-MUSIC.

If the contours on the gain surface corresponding to a fixed value of $\alpha$ is traced in the direction of increasing GSNR, the superiority of the FLOS-based estimator begins at a low GSNR threshold where the estimator becomes firstly effective. The increase in the gain continues along this contour until a peak value of the gain is reached. This behavior of the gain is dominated by the variance performance of the FLOS-Bartlett estimator shown in Fig. 4. Further increase in GSNR causes a reduction in the gain as the additive $\mathrm{S} \alpha \mathrm{S}$ noise becomes gradually ineffective. The reduction in the gain is a consequence of the increased ability of the SOS-based estimator in modeling the process. Tracing a contour of gain surface for GSNR $=10 \mathrm{~dB}$ shows that the gain increases in the direction of decreasing $\alpha$ which is caused by the fading ability of the SOS-based methods as the impulsiveness increases.

\subsubsection{Frequency dependence of bias and variance}

The sample variance, and the bias of the MUSIC and FLOS-MUSIC frequency estimators are displayed against the angular frequency for $\alpha=1.001$ and GSNR $=5 \mathrm{~dB}$ in Fig. 3. The number of Monte Carlo runs is 1000 , each with a different noise and phase realization. The FLOS-MUSIC has approximately $5 \mathrm{~dB}$ lower sample variance than the MUSIC.

The bias curves depict a symmetry around approximately $\omega=1.57 \mathrm{rad} / \mathrm{s}$. The FLOS-MUSIC performs much better than the MUSIC. The difference of their bias value is more than $0.4 \mathrm{rad} / \mathrm{s}$ around $\omega=0.2 \mathrm{rad} / \mathrm{s}$.

\subsubsection{Dependence of variance upon GSNR}

We utilize FLOS-based MUSIC, Bartlett, TK and MN estimators in this subsection. All of these frequency estimators have SOS-based versions whose variances change linearly and inversely in the signal-to-noise ratio in Gaussian noise environments. Fig. 4 depicts the dependence of the variance of the FLOS-based estimators upon GSNR for $\alpha=1.001$. The curves reflect averages over different frequencies. The variance level of $-40 \mathrm{~dB}$ observed around GSNR $=30 \mathrm{~dB}$ is maintained by the FLOS-TK and FLOS-MN frequency estimators even when the GSNR is increased unboundedly. This fact is caused by the error floor present in these frequency estimators since they both have a FLOS quantity multiplied by another FLOS quantity. The error floor for the FLOS-Bartlett frequency estimator is at a level of $-80 \mathrm{~dB}$ which is attained around GSNR $=50 \mathrm{~dB}$. The 

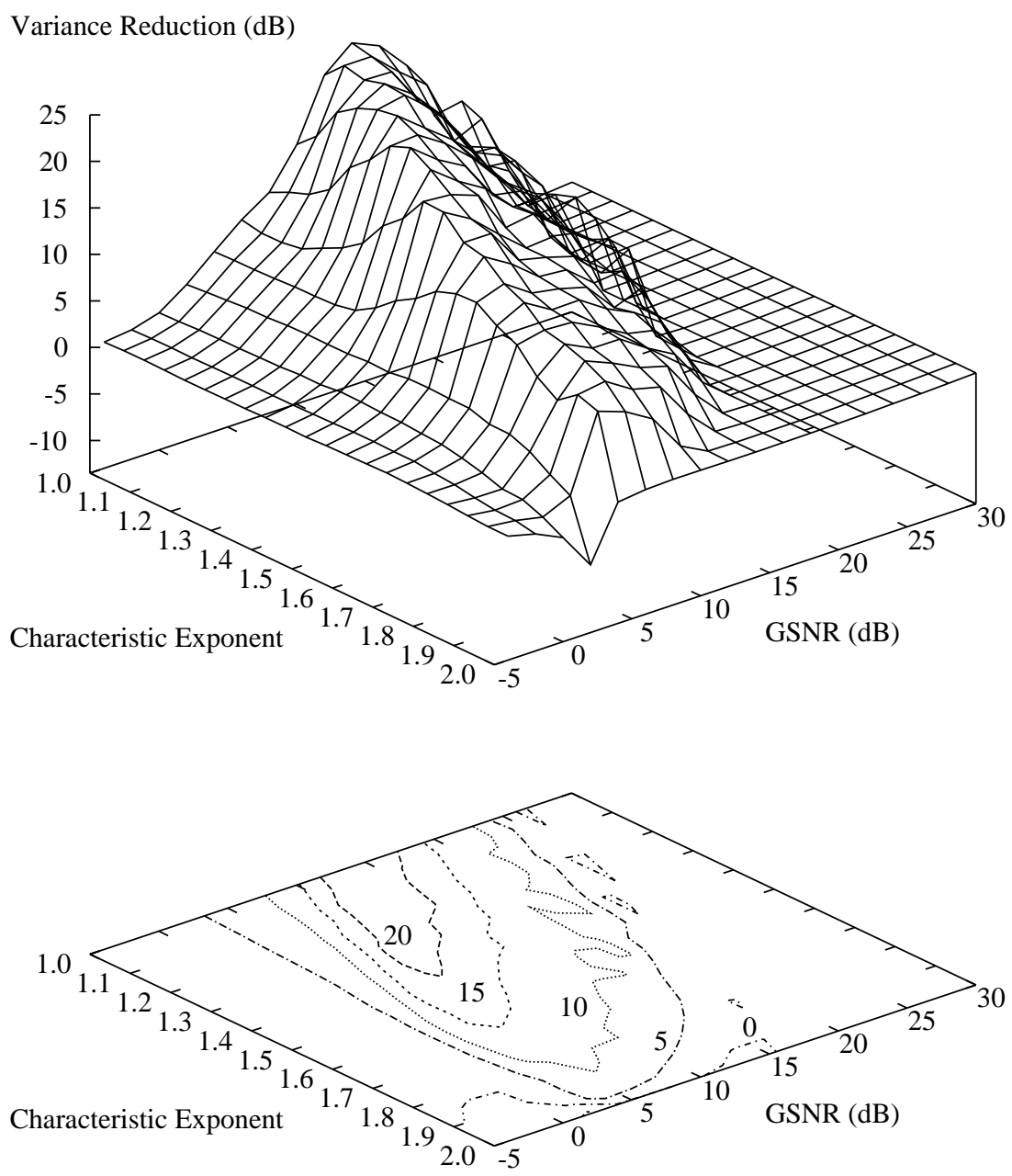

Fig. 2. Variance reduction of FLOS-Bartlett with respect to PC-Bartlett frequency estimator versus GSNR and characteristic exponent of alpha-stable noise, averaged on the frequency axis $(M=20, N=50,100$ noise and phase realizations).

reason for this behavior is the deviation of the estimated signal-subspace eigenvalues from their theoretically equal values. On the other hand, FLOS-MUSIC's variance changes linearly and inversely in GSNR.

\subsubsection{Dependence of bias upon $\alpha$}

The bias behavior of the estimators for $\omega=0.76 \mathrm{rad} / \mathrm{s}$ as a function of the characteristic exponent $\alpha$ of the noise is shown in Fig. 5. The figure indicates that the bias gets smaller as $\alpha$ increases. When $\alpha=1.001$, the bias values are greater than $0.3 \mathrm{rad} / \mathrm{s}$ for PC-Bartlett, MUSIC and TK, whereas it is less than $0.1 \mathrm{rad} / \mathrm{s}$ for their FLOS versions (except for FLOS-TK, which is slightly above $0.1 \mathrm{rad} / \mathrm{s}$ for $1.001 \leqslant \alpha \leqslant 1.06$ ). Note that the bias of FLOS-TK decreases slowly and exceeds that of the TK estimator for $\alpha>1.6$, which is not a phenomenon encountered with the other methods. The bias behavior of the FLOS-TK frequency estimator can be explained by the fact that it is based on a product of two FLOS quantities. For the single tone case as in our experiments, MUSIC and PC-Bartlett estimators show exactly the same performance. Similarly, 

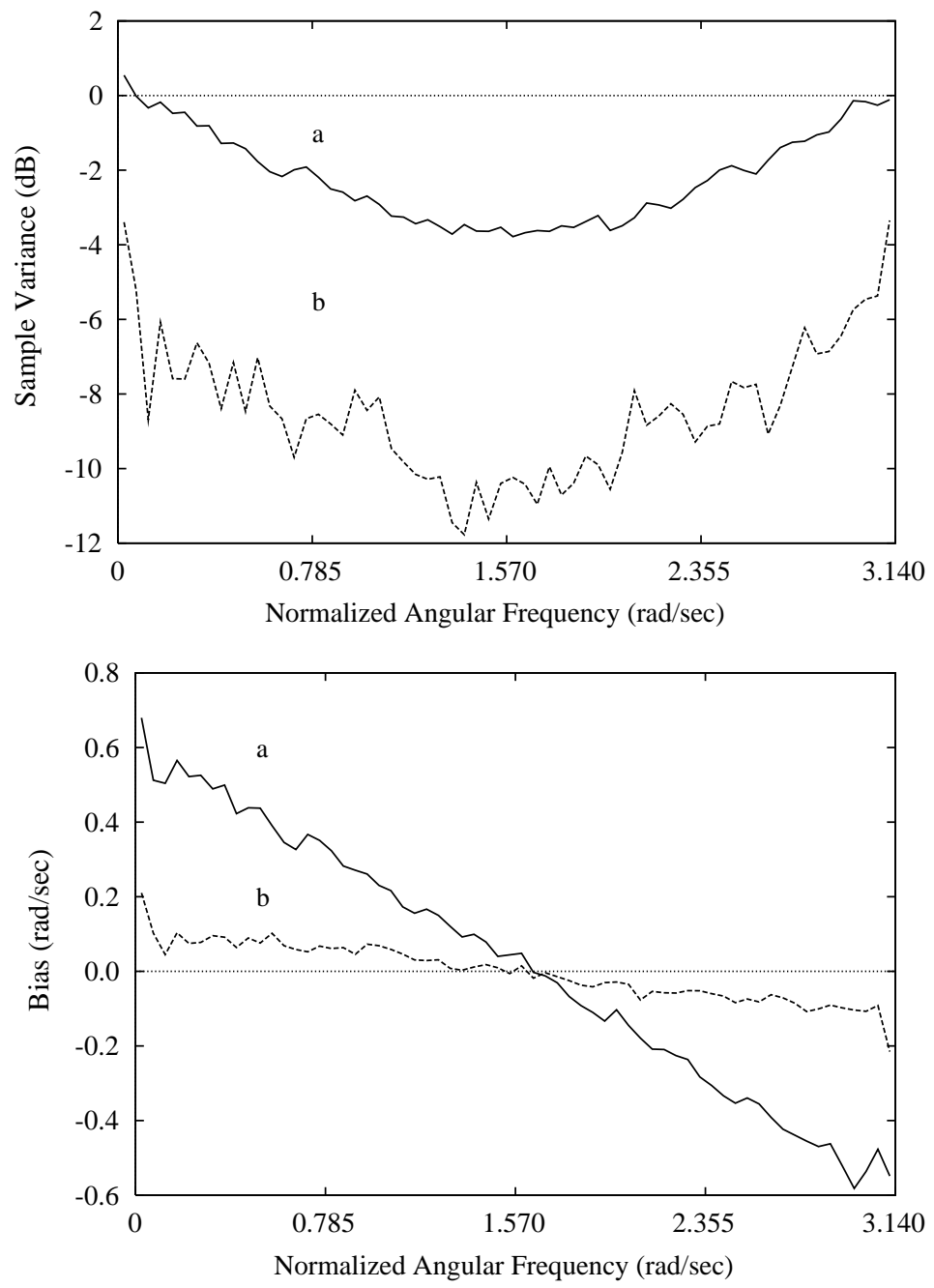

Fig. 3. Sample variance and bias of MUSIC and FLOS-MUSIC frequency estimators versus normalized angular frequency: (a) MUSIC, (b) FLOS-MUSIC ( $\alpha=1.001, p=1$. (FLOS-MUSIC), $M=20$, GSNR $=5 \mathrm{~dB}, N=50,1000$ noise and phase realizations).

FLOS-MUSIC and FLOS-Bartlett estimators exhibit the same performance just like their SOS-based versions, at least for GSNR values smaller than $35 \mathrm{~dB}$.

\subsection{Two real tones case}

In each Monte Carlo run, different additive $\mathrm{S} \alpha \mathrm{S}$ noise and phase realizations are applied. The moment order for the GCC estimate is chosen as 1 , and the size of the GCC matrix is $20 \times 20$.

\subsubsection{Dependence of resolution probability upon GSNR and $\alpha$}

The subspace techniques are favored in sinusoidal parameter estimation problems due to their high-resolution probabilities that is their high ability to distinguish two signals closely spaced in frequency. An easy-to-obtain 


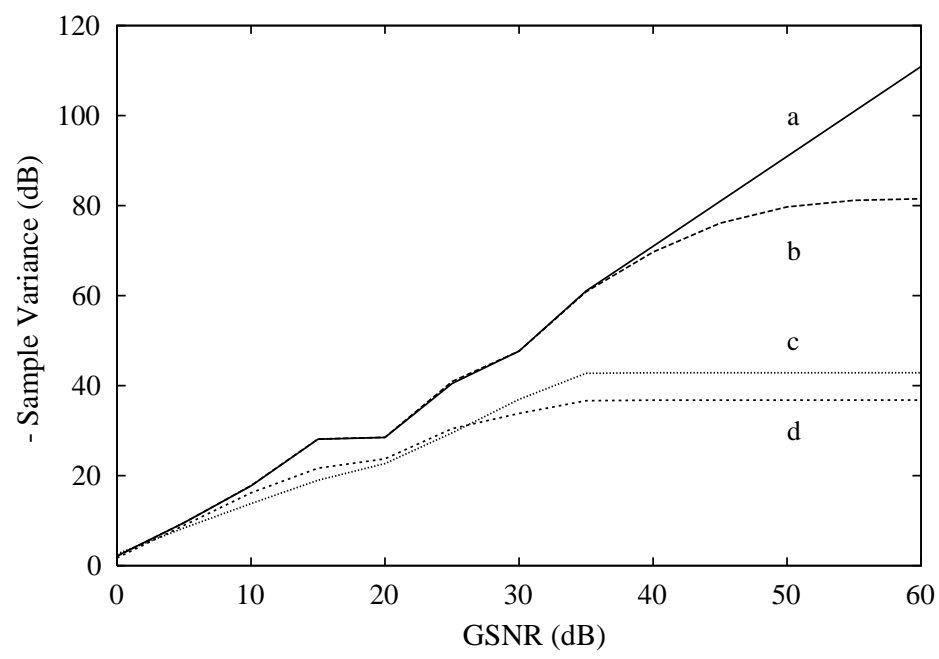

Fig. 4. Sample variance of FLOS-based frequency estimators versus GSNR averaged on the frequency axis: (a) FLOS-MUSIC, (b) FLOS-Bartlett, (c) FLOS-TK, and (d) FLOS-MN $(\alpha=1.001, M=20, N=50,1000$ noise and phase realizations).

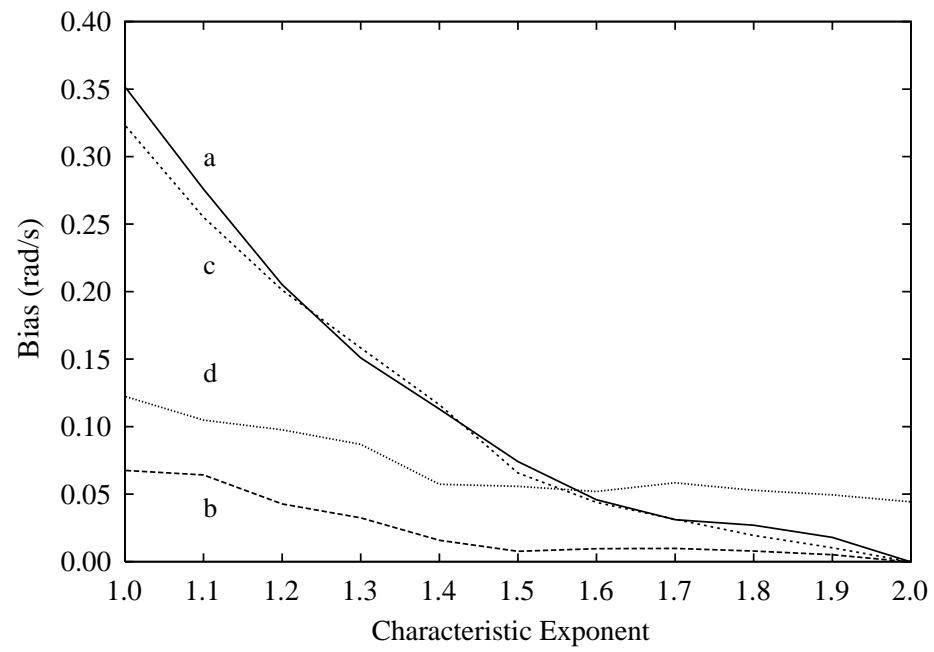

Fig. 5. Bias of second-order statistics based and FLOS-based frequency estimators versus characteristic exponent of alpha-stable noise: (a) PC-Bartlett and MUSIC, (b) FLOS-Bartlett and FLOS-MUSIC, (c) TK, and (d) FLOS-TK ( $\omega=0.76 \mathrm{rad} / \mathrm{s}, M=20$, GSNR $=5 \mathrm{~dB}$, $N=50,1000$ noise and phase realizations).

and good measure of the resolution probability is found using the resolution event given by the random inequality [20]

$$
\rho\left(f_{1}, f_{2}\right) \doteq P\left(f_{m}\right)-\frac{1}{2}\left\{P\left(f_{1}\right)+P\left(f_{2}\right)\right\}<0,
$$

where $f_{1}, f_{2}$ are the sinusoidal frequencies and $f_{m}=\left(f_{1}+f_{2}\right) / 2$ denotes their mean. $P(f)$ is the power spectrum of the estimator, which means that the sinusoidal frequencies correspond to the peaks of $P(f)$ and 


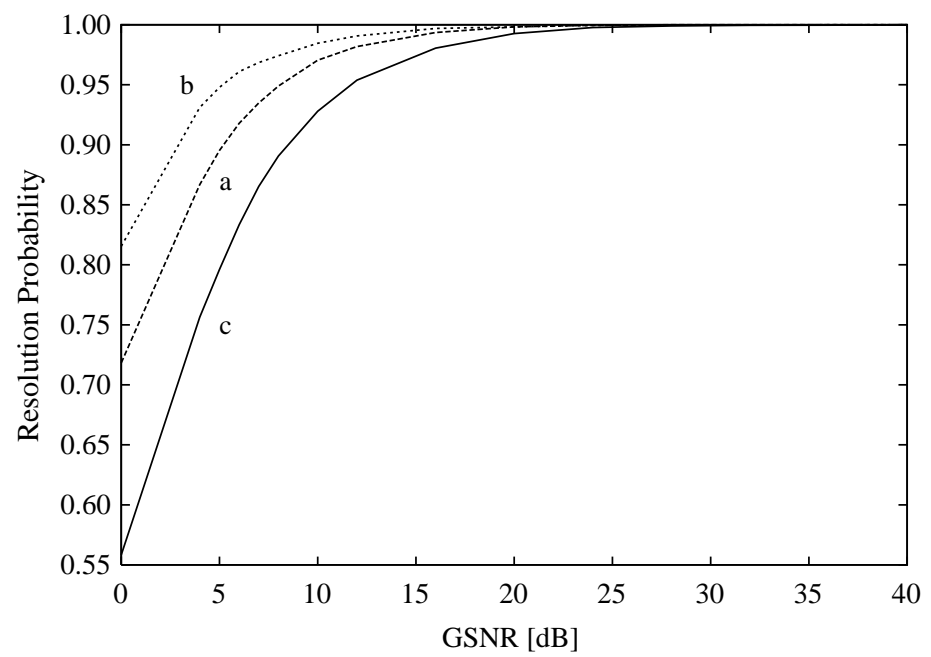

Fig. 6. Resolution probabilities of MUSIC, FLOS-MUSIC and periodogram frequency estimators versus GSNR: (a) MUSIC, (b) FLOS-MUSIC, and (c) periodogram $\left(\alpha=1.001, \omega_{1}=1.57 \mathrm{rad} / \mathrm{s}, \omega_{2}-\omega_{1}=0.503 \mathrm{rad} / \mathrm{s}, M=20, N=50,10000\right.$ noise and phase realizations).

$\rho$ is the decision statistics. The sinusoidal frequencies are resolved when the inequality holds. The resolution probability is found as the ratio of the simulation runs when $\rho$ is true and the total number of simulation runs. In Fig. 6 the resolution probabilities of MUSIC and FLOS-MUSIC frequency estimators are plotted against the GSNR. Also the resolution probability of the periodogram frequency estimator is shown in the figure for comparison. It is well known that the resolution limit of Fourier methods is approximately an angular frequency difference of $2 \pi / N$ for sinusoidal signals observed in Gaussian noise [4,8]. In the simulation, we use $\alpha=1.001$ and $N=50$. Even though the gap of the sinusoidal frequencies is as much as four times the Fourier resolution limit, the resolution probability of the periodogram method is worse than the resolution probabilities of MUSIC and FLOS-MUSIC. At GSNR $=5 \mathrm{~dB}$, FLOS-MUSIC resolves sinusoidal frequencies nearly $95 \%$ of the time, whereas this chance is approximately $90 \%$ and $80 \%$ for the MUSIC and periodogram estimators, respectively, which is also shown in Fig. 7. The relative advantage of FLOS-MUSIC with respect to MUSIC is as much as $10 \%$ at GSNR $=0 \mathrm{~dB}$.

In Fig. 7, the resolution probabilities of the FLOS-MUSIC, MUSIC and the periodogram estimators are plotted against $\alpha$ for GSNR $=5 \mathrm{~dB}$. All of the estimators attain the resolution probability one at $\alpha=2$ while their performance get worse as $\alpha$ decreases. Although the performance of MUSIC is better than the periodogram estimator, the relative advantage of FLOS-MUSIC with respect to MUSIC gets larger and finally reaches a value of $5 \%$ at $\alpha=1.001$. Hence, FLOS-based estimators tolerate a higher degree of impulsiveness in noise (represented by lower $\alpha$ values) compared to the MUSIC algorithm.

We also perform simulations with frequency difference less than the Fourier resolution limit. Both FLOSMUSIC and MUSIC attain nearly 100\% resolution at high GSNR values, e.g. at GSNR $=40 \mathrm{~dB}$ for $\alpha=1.001$. The superiority of FLOS-MUSIC over MUSIC disappears since the effect of noise becomes negligible at high GSNR values. Although the resolution probability of FLOS-MUSIC is higher than the one of MUSIC in those simulations performed at low GSNR values, the sinusoids are only resolved when their frequency difference is greater than the Fourier resolution limit in Gaussian noise. One can thus deduce that FLOS-based methods offer a significant advantage at relatively low GSNR values in non-high-resolution requiring scenarios. 


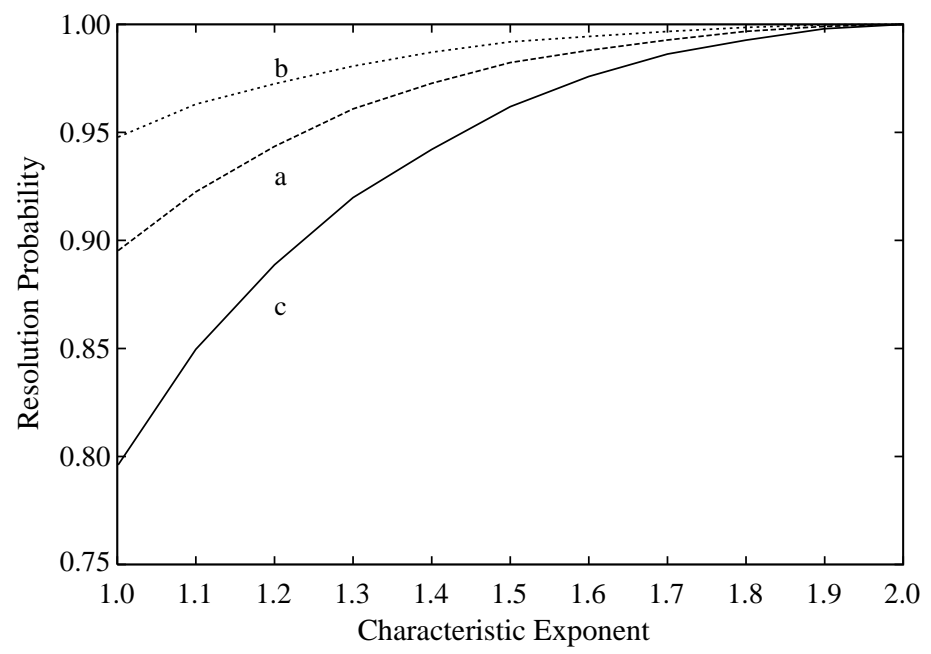

Fig. 7. Resolution probabilities of MUSIC, FLOS-MUSIC and periodogram frequency estimators versus the characteristic exponent of alpha-stable noise: (a) MUSIC, (b) FLOS-MUSIC, and (c) periodogram (GSNR $=5 \mathrm{~dB}, \omega_{1}=1.57 \mathrm{rad} / \mathrm{s}, \omega_{2}-\omega_{1}=0.503 \mathrm{rad} / \mathrm{s}, M=20$, $N=50,10000$ noise and phase realizations).

\subsubsection{Frequency dependence of bias and variance}

In Fig. 8, the sample variance and bias of the MUSIC and FLOS-MUSIC frequency estimators for the varying sinusoidal frequency are plotted against the angular frequency difference between the tone frequencies for $\alpha=1.001$ and GSNR $=2 \mathrm{~dB}$. We fix the angular frequency of one sinusoid at $\omega=\pi / 2 \mathrm{rad} / \mathrm{s}$. In this experiment, the sample size is 1000 and the number of noise and phase realizations is 200. The FLOSMUSIC has approximately $3 \mathrm{~dB}$ less sample variance than the SOS-based MUSIC.

The bias curve shows symmetry around the angular frequency difference $\omega_{2}-\omega_{1}=0 \mathrm{rad} / \mathrm{s}$. The FLOSMUSIC performs better than MUSIC. The difference of their bias value is approximately $0.3 \mathrm{rad} / \mathrm{s}$ around $\omega_{2}-\omega_{1}=0.25 \mathrm{rad} / \mathrm{s}$.

\section{Conclusion}

We have proposed a FLOS-based quantity, the GCC, where no assumptions are made on the pdf of the random variables to which it is applied. For moment order $p=1$, the GCC matrix of multiple sinusoids in $\mathrm{S} \alpha \mathrm{S}$ noise attains the same form as that of the covariance matrix of multiple sinusoids in additive white Gaussian noise which results in the best performance of the FLOS-based subspace techniques considering other choices for the $p$ value. The obvious advantage of the GCC matrix with respect to the covariance matrix is that its elements are bounded for data containing $\mathrm{S} \alpha \mathrm{S}$ components as long as $p<\alpha$. Thus, the FLOS-based versions of the well-known MUSIC and PC-Bartlett frequency estimators can be applied in multiple sinusoids in $\mathrm{S} \alpha \mathrm{S}$ noise scenarios.

When the GCC of two jointly $\mathrm{S} \alpha \mathrm{S}$ distributed random variables is formed, the GCC reduces to the covariation coefficient, hence the name generalized covariation coefficient. We also showed that the GCCs can be used when the multiple sinusoids in $\mathrm{S} \alpha \mathrm{S}$ noise are modeled as a stable ARMA process approximated by a higher order stable AR process. The frequency estimators FLOS-TK and FLOS-MN are based on this model.

Rigorous simulations showed that when the additive noise in the frequency estimation problem can be modeled as an alpha-stable process, the FLOS-based subspace techniques perform better than their SOS-based 

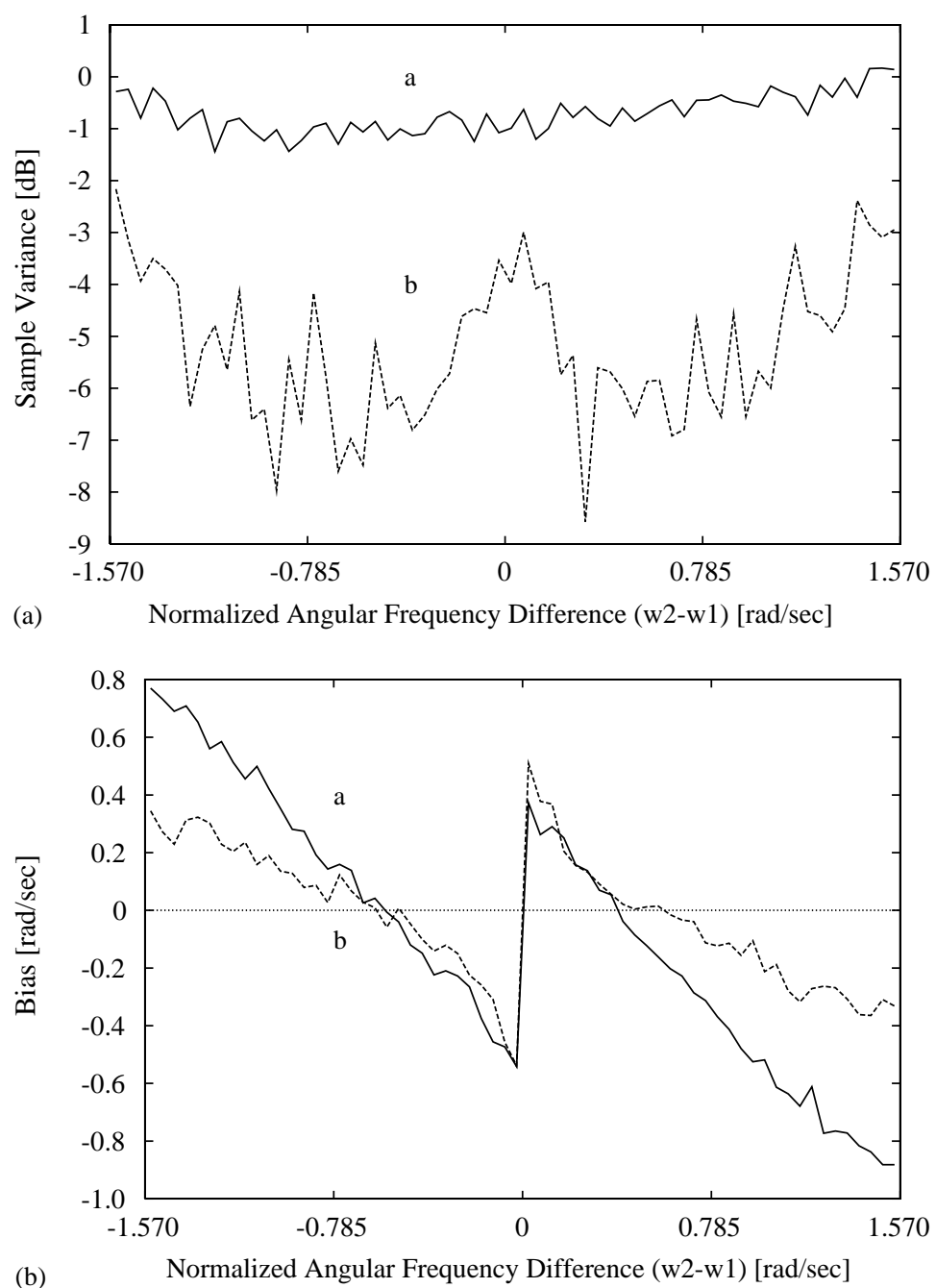

Fig. 8. Sample variance and bias of MUSIC and FLOS-MUSIC frequency estimators for the varying sinusoidal frequency versus normalized angular frequency difference: (a) MUSIC, and (b) FLOS-MUSIC $\left(\alpha=1.001, p=1.0\left(\right.\right.$ FLOS-MUSIC), $\omega_{1}=1.57 \mathrm{rad} / \mathrm{s}$, $M=20, \mathrm{GSNR}=2 \mathrm{~dB}, N=1000,200$ noise and phase realizations).

counterparts. This outcome is particularly true for the impulsive noise described by low $\alpha$ values when the SOS-based subspace techniques lose their ability to model the system due to unbounded statistics.

In the case of a single sinusoidal signal both FLOS-MUSIC and FLOS-Bartlett not only demonstrate superior performance over MUSIC and PC-Bartlett for low $\alpha$ values, but they are at the same time robust enough to perform comparably with the SOS-based methods in the presence of non-impulsive noise. The TK and $\mathrm{MN}$ estimators perform considerably better than their FLOS versions in non-impulsive noise, negating any robustness claims. Thus, FLOS-MUSIC and FLOS-Bartlett must be favored if satisfactory results are desired regardless of the noise distribution.

In the frequency estimation of multiple sinusoids, the estimators which demonstrate robust performance irrespective of the characteristic exponent of the $\mathrm{S} \alpha \mathrm{S}$ noise in the experiments with a single sinusoid, namely 
FLOS-MUSIC and FLOS-Bartlett are investigated. In the case of multiple sinusoids, a very important issue is whether the sinusoids are well resolved or not. Both FLOS-Bartlett and PC-Bartlett failed in the resolution experiments. On the contrary FLOS-MUSIC and MUSIC were successful. While FLOS-MUSIC and MUSIC showed comparable high-resolution capability at high GSNR values, FLOS-MUSIC offers a significant advantage at relatively low GSNR values in non-high-resolution requiring scenarios.

Still open issues include the development of implementable blind estimators, and techniques that function in colored alpha-stable noise.

\section{Appendix A. The GCCs of a single sinusoid in $\mathrm{S} \alpha \mathrm{S}$ noise}

Assume that the observation sequence $x_{n}$ is given by Eq. (9) where $K=1$. When $p=1$ the GCC of $X_{n}$ with $X_{l}$ is defined as

$$
\begin{aligned}
\left.\lambda_{X_{n}, X_{l}}(p)\right|_{p=1} & =E\left\{\left(A_{1} \sin \left\{\omega_{1} n+\Theta_{1}\right\}+Z_{n}\right) \operatorname{signum}\left(A_{1} \sin \left\{\omega_{1} l+\Theta_{1}\right\}+Z_{l}\right)\right\}\left(E\left|A_{1} \sin \left\{\omega_{1} l+\Theta_{1}\right\}+Z_{l}\right|\right)^{-1} \\
& =\frac{\operatorname{NOM}_{n-l}^{(1)}}{\operatorname{DENOM}_{n-l}^{(1)}} .
\end{aligned}
$$

The nominator in Eq. (A.1) can be written as

$$
\begin{aligned}
\operatorname{NOM}_{n-l}^{(1)}= & \frac{1}{2 \pi} \int_{0}^{2 \pi} \int_{-\infty}^{+\infty} \int_{-\infty}^{+\infty}\left\{\left(A_{1} \sin \left\{\omega_{1} n+\theta_{1}\right\}+z_{n}\right) \operatorname{signum}\left(A_{1} \sin \left\{\omega_{1} l+\theta_{1}\right\}+z_{l}\right)\right. \\
& \left.\times f_{Z_{n}, Z_{l}}\left(z_{n}, z_{l}\right)\right\} \mathrm{d} z_{n} \mathrm{~d} z_{l} \mathrm{~d} \theta_{1},
\end{aligned}
$$

where $f_{Z_{n}, Z_{l}}\left(z_{n}, z_{l}\right)$ is the joint pdf of the $\mathrm{S} \alpha \mathrm{S}$ noise samples $Z_{n}$ and $Z_{l}$. With the change of variable $\phi_{1}=$ $\omega_{1} l+\theta_{1}$, one obtains

$$
\begin{aligned}
\mathrm{NOM}_{n-l}^{(1)}= & \frac{1}{2 \pi} \int_{0}^{2 \pi} \int_{-\infty}^{+\infty} \int_{-\infty}^{+\infty}\left\{\left(A_{1} \sin \left\{\omega_{1}(n-l)+\phi_{1}\right\}+z_{n}\right) \operatorname{signum}\left(A_{1} \sin \phi_{1}+z_{l}\right)\right. \\
& \left.\times f_{Z_{n}, Z_{l}}\left(z_{n}, z_{l}\right)\right\} \mathrm{d} z_{n} \mathrm{~d} z_{l} \mathrm{~d} \phi_{1} .
\end{aligned}
$$

Since noise samples are zero-mean, independent and identically distributed, one can find the expression in the previous equation for $n \neq l$ as

$$
\left.\mathrm{NOM}_{n-l}^{(1)}\right|_{n \neq l}=\frac{1}{2 \pi} \int_{0}^{2 \pi} \int_{-\infty}^{+\infty} A_{1} \sin \left\{\omega_{1}(n-l)+\phi_{1}\right\} \operatorname{signum}\left(A_{1} \sin \phi_{1}+z_{l}\right) f_{Z_{l}}\left(z_{l}\right) \mathrm{d} z_{l} \mathrm{~d} \phi_{1} .
$$

In order to evaluate this double integral we substitute

$$
\operatorname{signum}\left(A_{1} \sin \phi_{1}+z_{l}\right)=\left\{\begin{aligned}
1 & \text { for } z_{l}>-A_{1} \sin \phi_{1} \\
0 & \text { for } z_{l}=-A_{1} \sin \phi_{1}, \\
-1 & \text { for } z_{l}<-A_{1} \sin \phi_{1} .
\end{aligned}\right.
$$


Thus, we obtain

$$
\begin{aligned}
\left.\mathrm{NOM}_{n-l}^{(1)}\right|_{n \neq l}= & \frac{1}{2 \pi}\left\{\int_{0}^{2 \pi}-A_{1} \sin \left\{\omega_{1}(n-l)+\phi_{1}\right\} \int_{-\infty}^{-A_{1} \sin \phi_{1}} f_{Z_{l}}\left(z_{l}\right) \mathrm{d} z_{l} \mathrm{~d} \phi_{1}\right. \\
& \left.+\int_{0}^{2 \pi} A_{1} \sin \left\{\omega_{1}(n-l)+\phi_{1}\right\} \int_{-A_{1} \sin \phi_{1}}^{+\infty} f_{Z_{l}}\left(z_{l}\right) \mathrm{d} z_{l} \mathrm{~d} \phi_{1}\right\} .
\end{aligned}
$$

Using trigonometric identities

$$
\begin{aligned}
\left.\operatorname{NOM}_{n-l}^{(1)}\right|_{n \neq l}=\frac{1}{2 \pi}[- & \cos \left\{\omega_{1}(n-l)\right\} \int_{0}^{2 \pi} A_{1} \sin \phi_{1} F_{Z_{l}}\left(-A_{1} \sin \phi_{1}\right) \mathrm{d} \phi_{1}+\sin \left\{\omega_{1}(n-l)\right\} \\
& \left.\times \int_{0}^{2 \pi} A_{1} \cos \phi_{1} F_{Z_{l}}\left(-A_{1} \sin \phi_{1}\right) \mathrm{d} \phi_{1}\right) \\
& +\left(\cos \left\{\omega_{1}(n-l)\right\} \int_{0}^{2 \pi} A_{1} \sin \phi_{1}\left[1-F_{Z_{l}}\left(-A_{1} \sin \phi_{1}\right)\right] \mathrm{d} \phi_{1}\right. \\
& \left.\left.+\sin \left\{\omega_{1}(n-l)\right\} \int_{0}^{2 \pi} A_{1} \sin \phi_{1}\left[1-F_{Z_{l}}\left(-A_{1} \sin \phi_{1}\right)\right] \mathrm{d} \phi_{1}\right)\right]
\end{aligned}
$$

and this reduces to

$$
\left.\mathrm{NOM}_{n-l}^{(1)}\right|_{n \neq l}=\frac{I_{1}}{\pi} \cos \left\{\omega_{1}(n-l)\right\},
$$

where

$$
I_{1}=\int_{0}^{2 \pi}-A_{1} \sin \phi_{1} F_{Z_{l}}\left(-A_{1} \sin \phi_{1}\right) \mathrm{d} \phi_{1}
$$

and $F_{Z_{l}}(\cdot)$ is the cumulative density function (cdf) of the random variable $Z_{l}$. It is easy to see that for any symmetric pdf $I_{1}$ is a positive constant whose value depends on the particular $\mathrm{S} \alpha \mathrm{S}$ pdf and on the magnitude of the sinusoidal signal.

For the case of $n=l$, we can write the nominator in Eq. (A.1) as $\operatorname{NOM}_{n-l}^{(1)}=\left(I_{1} / \pi\right)+P_{z_{l}}^{\prime(1)}$ where

$$
\begin{aligned}
P_{z_{l}}^{\prime(1)} & =\frac{1}{2 \pi} \int_{0}^{2 \pi} \int_{-\infty}^{+\infty} z_{l} \operatorname{signum}\left(A_{1} \sin \phi_{1}+z_{l}\right) f_{Z_{l}}\left(z_{l}\right) \mathrm{d} z_{l} \mathrm{~d} \phi_{1} \\
& =\frac{1}{\pi} \int_{0}^{2 \pi} \int_{-A_{1} \sin \phi_{1}}^{+\infty} z_{l} f_{Z_{l}}\left(z_{l}\right) \mathrm{d} z_{l} \mathrm{~d} \phi_{1}
\end{aligned}
$$

denotes the term due to the additive $\mathrm{S} \alpha \mathrm{S}$ noise. $P_{z_{l}}^{\prime(1)}$ is a positive, finite quantity for any $\mathrm{S} \alpha \mathrm{S}$ pdf satisfying $1<\alpha \leqslant 2$.

One can easily see that

$$
\operatorname{DENOM}_{n-l}^{(1)}=E\left|A_{1} \sin \left\{\omega_{1} l+\Theta_{1}\right\}+Z_{l}\right|
$$

is also a positive constant. 
Thus, for the unity moment order, the GCCs of a single sinusoid observed in $\mathrm{S} \alpha \mathrm{S}$ noise can be expressed as

$$
\left.\lambda_{X_{n}, X_{l}}(p)\right|_{p=1}=\zeta_{1} \cos \left\{\omega_{1}(n-l)\right\}+P_{z_{l}}^{(1)} \delta_{n l},
$$

where $\zeta_{1}$ is a positive real constant given as $\zeta_{1}=I_{1} /\left(\pi \mathrm{DENOM}_{n-l}^{(1)}\right), P_{z_{l}}^{(1)}$ is given as $P_{z_{l}}^{(1)}=P_{z_{l}}^{\prime(1)} / \mathrm{DENOM}_{0}^{(1)}$ and $\delta_{n l}$ is the Kronecker delta.

\section{Appendix B. The GCCs of two sinusoids in $\mathrm{S} \alpha \mathrm{S}$ noise}

The GCCs of $X_{n}$ with $X_{l}$ described by Eq. (9) where $K=2$ can be written for $p=1$ as

$$
\begin{aligned}
\left.\lambda_{X_{n}, X_{l}}(p)\right|_{p=1}= & E\left\{\left(\sum_{i=1}^{2}\left[A_{i} \sin \left\{\omega_{i} n+\Theta_{i}\right\}\right]+Z_{n}\right) \operatorname{signum}\left(\sum_{j=1}^{2}\left[A_{j} \sin \left\{\omega_{j} l+\Theta_{j}\right\}\right]+Z_{l}\right)\right\} \\
& \times\left(E\left|\sum_{j=1}^{2}\left[A_{j} \sin \left\{\omega_{j} l+\Theta_{j}\right\}+Z_{l}\right]\right|\right)^{-1} \\
= & \frac{\operatorname{NOM}_{n-l}^{(2)}}{\operatorname{DENOM}_{n-l}^{(2)}} .
\end{aligned}
$$

With a change of variables $\phi_{i}=\omega_{i} l+\theta_{i}$ for $i=1,2$, one obtains

$$
\begin{aligned}
\operatorname{NOM}_{n-l}^{(2)}= & \left(\frac{1}{2 \pi}\right)^{2} \int_{0}^{2 \pi} \int_{0}^{2 \pi} \int_{-\infty}^{+\infty} \int_{-\infty}^{+\infty}\left\{\left(\sum_{i=1}^{2}\left[A_{i} \sin \left\{\omega_{i}(n-l)+\phi_{i}\right\}\right]+z_{n}\right)\right. \\
& \left.\times \operatorname{signum}\left(\sum_{j=1}^{2}\left[A_{j} \sin \left\{\phi_{j}\right\}\right]+z_{l}\right) f_{Z_{n}, Z_{l}}\left(z_{n}, z_{l}\right)\right\} \mathrm{d} z_{n} \mathrm{~d} z_{l} \mathrm{~d} \phi_{1} \mathrm{~d} \phi_{2} .
\end{aligned}
$$

We partition this integral to two parts where the arguments of the signum( $(\cdot)$ function are positive or negative. Hence, the right-hand side of Eq. (B.2) for the case of $n \neq l$ can be written as

$$
\begin{aligned}
\left.\mathrm{NOM}_{n-l}^{(2)}\right|_{n \neq l}= & \left(\frac{1}{2 \pi}\right)^{2}\left\{\left(\int_{0}^{2 \pi} \int_{0}^{2 \pi}-\sum_{i=1}^{2}\left[A_{i} \sin \left\{\omega_{i}(n-l)+\phi_{i}\right\}\right] \int_{-\infty}^{-\sum_{i=1}^{2} A_{i} \sin \phi_{i}} f_{Z_{l}}\left(z_{l}\right) \mathrm{d} z_{l} \mathrm{~d} \phi_{1} \mathrm{~d} \phi_{2}\right)\right. \\
& \left.+\int_{0}^{2 \pi} \int_{0}^{2 \pi} \sum_{i=1}^{2}\left[A_{1} \sin \left\{\omega_{1}(n-l)+\phi_{1}\right\}\right] \int_{-\sum_{i=1}^{2} A_{i} \sin \phi_{i}}^{+\infty} f_{Z_{l}}\left(z_{l}\right) \mathrm{d} z_{l} \mathrm{~d} \phi_{1} \mathrm{~d} \phi_{2}\right\} .
\end{aligned}
$$

Again using trigonometric identities and procedures similar to those followed in Appendix A, one obtains

$$
\begin{aligned}
\left.\mathrm{NOM}_{n-l}^{(2)}\right|_{n \neq l}= & \frac{1}{2 \pi^{2}} \cos \left\{\omega_{1}(n-l)\right\} \int_{0}^{2 \pi} \int_{0}^{2 \pi}-A_{1} \sin \phi_{1} F_{Z_{l}}\left(\sum_{i=1}^{2}-A_{i} \sin \phi_{i}\right) \mathrm{d} \phi_{2} \mathrm{~d} \phi_{1} \\
& +\frac{1}{2 \pi^{2}} \cos \left\{\omega_{2}(n-l)\right\} \int_{0}^{2 \pi} \int_{0}^{2 \pi}-A_{2} \sin \phi_{2} F_{Z_{l}}\left(\sum_{i=1}^{2}-A_{i} \sin \phi_{i}\right) \mathrm{d} \phi_{1} \mathrm{~d} \phi_{2} .
\end{aligned}
$$


Similar to the case of Appendix A, the double integrals in Eq. (B.4) are positive constants whose values depend on the particular $\mathrm{S} \alpha \mathrm{S}$ pdf, $f_{Z_{l}}$, and on the magnitudes of the two sinusoids.

For the case of $n=l$, the nominator of the right-hand side of Eq. (B.1), $\left.\mathrm{NOM}_{n-l}^{(2)}\right|_{n=l}$, becomes the sum of two parts. The first one which we can call as the signal part, is obtained by setting $n=l$ in the right-hand side of Eq. (B.4). The second part resulting from the $\mathrm{S} \alpha \mathrm{S}$ noise can be calculated as

$$
\begin{aligned}
P_{z_{l}}^{\prime(2)} & =\left(\frac{1}{2 \pi}\right)^{2} \int_{0}^{2 \pi} \int_{0}^{2 \pi} \int_{-\infty}^{+\infty} z_{l} \operatorname{signum}\left(\sum_{i=1}^{2}\left[A_{1} \sin \phi_{1}\right]+z_{l}\right) f_{Z_{l}}\left(z_{l}\right) \mathrm{d} z_{l} \mathrm{~d} \phi_{1} \mathrm{~d} \phi_{2} \\
& =\frac{1}{2 \pi^{2}} \int_{0}^{2 \pi} \int_{0}^{2 \pi} \int_{-\sum_{i=1}^{2} A_{i} \sin \phi_{i}}^{+\infty} z_{l} f_{Z_{l}}\left(z_{l}\right) \mathrm{d} z_{l} \mathrm{~d} \phi_{1} \mathrm{~d} \phi_{2}
\end{aligned}
$$

which is again a positive finite constant for any $\mathrm{S} \alpha \mathrm{S}$ pdf with $1<\alpha \leqslant 2$.

The denominator of the right-hand side of Eq. (B.1) given by

$$
\operatorname{DENOM}_{n-l}^{(2)}=E\left|\sum_{j=1}^{2}\left[A_{j} \sin \left\{\omega_{j} l+\Theta_{j}\right\}\right]+Z_{l}\right|
$$

is also a positive constant.

Thus, one can express the GCCs of two sinusoids in $\mathrm{S} \alpha \mathrm{S}$ noise for the moment order $p=1$ as

$$
\left.\lambda_{X_{n}, X_{l}}(p)\right|_{p=1}=\sum_{i=1}^{2} \xi_{i} \cos \left\{\omega_{i}(n-l)\right\}+P_{z_{l}}^{(2)} \delta_{n l},
$$

where $\xi_{1}$ and $\xi_{2}$ are positive real constants depending on the particular $\mathrm{S} \alpha \mathrm{S}$ pdf.

\section{References}

[1] S. Cambanis, G. Miller, Linear problems in pth order and stable processes, SIAM J. Appl. Math. 41 (1) (August 1981 ) $43-69$.

[2] J.M. Chambers, C.L. Mallows, B.W. Stuck, A method for simulating stable random variables, J. Amer. Statist. Assoc. 71 (354) (June 1976) 340-344.

[3] J. Friedmann, H. Messer, J.-F. Cardoso, Robust parameter estimation of a deterministic signal in impulsive noise, IEEE Trans. Signal Process. 48 (4) (April 2000) 935-942.

[4] S.M. Kay, Modern Spectral Estimation: Theory and Applications, Prentice-Hall, Englewood Cliffs, NJ, 1988.

[5] S. Kay, S.L. Marple, Spectrum analysis - a modern perspective, Proc. IEEE 69 (11) (November 1981) $1380-1419$.

[6] T. Liu, J.M. Mendel, A subspace-based direction finding algorithm using fractional lower order statistics, IEEE Trans. Signal Process. 49 (8) (August 2001) 1605-1613.

[7] X. Ma, C.L. Nikias, Parameter estimation and blind channel identification in impulsive signal environment, IEEE Trans. Signal Process. 43 (12) (December 1995) 2884-2897.

[8] S.L. Marple, Digital Spectral Analysis with Applications, Prentice-Hall, Englewood Cliffs, NJ, 1987.

[9] G. Miller, Properties of certain symmetric stable distribution, J. Multivariate Analy. 8 (1978) (346-360).

[10] C.L. Nikias, M. Shao, Signal Processing with Alpha-Stable Distributions and Applications, Wiley, New York, NY, 1995.

[11] J.G. Proakis, C.M. Rader, F. Ling, C.L. Nikias, Advanced Digital Signal Processing, Macmillan, New York, NY, 1992.

[12] M. Shao, C.L. Nikias, Signal processing with fractional lower order moments: stable processes and their applications Proc. IEEE 81 (7) (July 1993) 986-1010.

[13] P. Stoica, R. Moses, Introduction to Spectral Analysis, Prentice-Hall, Upper Saddle River, NJ, 1997.

[14] P. Stoica, T. Söderström, F. Ti, Asymptotic properties of the high-order Yule-Walker estimates of sinusoidal frequencies, IEEE Trans. Acoust. Speech Signal Process. 37 (11) (November 1989) 1721-1734.

[15] A. Swami, B. Sadler, Parameter estimation for linear alpha-stable processes, IEEE Signal Process. Lett. 5 (2) (February 1998) 48-50.

[16] P. Tsakalides, C.L. Nikias, The robust covariation-based MUSIC (ROC-MUSIC) algorithm for bearing estimation in impulsive noise environments, IEEE Trans. Signal Process. 44 (7) (July 1996) 1623-1633. 
[17] G.A. Tsihrintzis, C.L. Nikias, Performance of optimum and suboptimum receivers in the presence of impulsive noise modeled as an alpha-stable process, IEEE Trans. Comm. 43 (2/3/4) (February/March/April 1995) 904-914.

[18] G.A. Tsihrintzis, C.L. Nikias, Fast estimation of the parameters of alpha-stable impulsive interference, IEEE Trans. Signal Process. 44 (6) (June 1996) 1492-1503.

[19] D.W. Tufts, R. Kumaresan, Estimation of frequencies of multiple sinusoids: making linear prediction perform like maximum likelihood Proc. IEEE 70 (9) (September 1982) 975-989.

[20] Q.T. Zhang, Probability of resolution of the MUSIC algorithm, IEEE Trans. Signal Process. 43 (4) (April 1995$) 978-987$. 